\title{
FREE PIECEWISE LINEAR INVOLUTIONS ON SPHERES
}

BY C. T. C. WALL

Communicated by W. Browder, November 14, 1967

If $T$ is a piecewise linear fixed-point free involution on $S^{n}$, the orbit space $Q^{n}=S^{n} / T$ is a PL-manifold homotopy equivalent to $P_{n}(R)=P^{n}$ [2]; the affirmative solution to the Poincare conjecture implies that conversely for $n \neq 3,4$ the double covering manifold of any such $Q^{n}$ can be identified with $S^{n}$. Write $I_{n}$ for the set of (oriented if $n$ is even) PL-homeomorphism classes of manifolds $Q^{n}$ homotopy equivalent to $P^{n}$. We will compute $I_{n}$ for $n \neq 3,4$.

Let $Q^{n}$ be as above. We define a normal invariant $\eta(Q)$. Take a homotopy equivalence $h: P^{n} \rightarrow Q^{n}$ (orientation-preserving if $n$ is odd): this is unique up to homotopy. Approximate $h \times 0$ by a PL-embedding $P^{n} \times Q^{n} \rightarrow R^{N}(N>n)$; let $\nu^{N}$ be the normal bundle of the embedding, which exists if $N$ is large enough [5], and $F: \nu^{N} \rightarrow \epsilon^{N}$ the fibre homotopy trivialisation induced by the homotopy equivalence [7], [10, 3.5]. Then $(\nu, F)$ induces a homotopy class $\eta(Q)$ of maps $P^{n} \rightarrow G / \mathrm{PL}$, which depends only on the PL-homeomorphism class of $Q$. We have thus defined $\eta: I_{n} \rightarrow\left[P^{n}, G / \mathrm{PL}\right]:$ our description follows Sullivan [8], the main idea goes back to Novikov [6].

We next compute $\left[P^{n}, G / \mathrm{PL}\right]$. The homotopy groups of $G / \mathrm{PL}$ are known to be $\boldsymbol{Z}$ (in dimensions $4 i$ ), $\boldsymbol{Z}_{2}$ (in dimensions $4 i+2$ ), and 0 (in odd dimensions). Further, Sullivan [8] has shown that if finite groups of odd order are ignored, the only nonzero $k$-invariant is the first (which is $\delta S q^{2}$ ). We choose fundamental classes $x_{2 i}$ $\in H^{2 i}\left(G / \mathrm{PL} ; \boldsymbol{Z}_{2}\right)(i \neq 2), \alpha \in H^{1}\left(P^{n} ; \boldsymbol{Z}_{2}\right)$. Because of the $k$-invariant, $\left[P^{4}, G / \mathrm{PL}\right] \cong Z_{4}$ : let $y$ be an isomorphism. Further, denote by $r$ the restriction $\left[P^{n+1}, G / \mathrm{PL}\right] \rightarrow\left[P^{n}, G / \mathrm{PL}\right]$. Then we have

Lemma 1. Let $i \geqq 0$. Then we have bijections

$$
\left[P^{2 i+5}, G / \mathrm{PL}\right] \stackrel{r}{\cong}\left[P^{2 i+4}, G / \mathrm{PL}\right] \stackrel{X}{\cong} Z_{4} \oplus \sum_{1 \leq j \leq i} z_{2},
$$

where the components of $X$ are $[y]=y r^{2 i}$ and $\left[x_{2 j+4}\right]$ with

$$
\left[x_{2 j+4}\right](f)=f^{*}\left(x_{2 j+4}\right) \alpha^{2 i-2 j}\left[P_{2 i+4}\right] \text {. }
$$

Moreover, $\left[x_{2}\right]$ is the mod 2 reduction of $[y]$.

We compute the image and 'kernel' of $\eta$ by surgery: in fact we have abelian groups $L_{n}\left(\boldsymbol{Z}_{2}^{+}\right)$and $L_{n}\left(\boldsymbol{Z}_{2}^{-}\right)$(the second referring to the non- 
orientable case) such that for $n \geqq 3$ the image of $\eta: I_{2 n} \rightarrow\left[P^{2 n}, G / \mathrm{PL}\right]$ is the kernel of a map $\vartheta$ to $L_{2 n}\left(Z_{\overline{2}}\right)$, and the fibres of $\eta$ are the orbits of an action of $L_{2 n+1}\left(Z_{2}\right)$ on $I_{2 n}$; similarly for $I_{2 n-1}$. These results will appear in [11]; also the following sharpening of a result of [9].

LEMMA 2. We have c: $L_{2 i}\left(Z_{\overline{2}}^{-}\right) \cong Z_{2}, L_{2 i+1}\left(Z_{2}^{-}\right)=0$, $(\sigma / 8, \tilde{\sigma} / 8): L_{4 i}\left(Z_{2}^{+}\right) \cong Z+Z, L_{4 i+1}\left(Z_{2}^{+}\right)=0, c: L_{4 i+2}\left(Z_{2}^{+}\right) \cong Z_{2}$

and $d: L_{4 i+3}\left(Z_{2}^{+}\right) \cong Z_{2}$, where $c$ denotes Kervaire-Arf invariant, $\sigma$ signature and $\tilde{\sigma}$ signature of double covers.

The next stage in the argument is the explicit computation of $c$. Results of Sullivan [8] and Browder, which are too long to summarize here, show that $c$ (see $[9,4.7]$ ) can be defined as an invariant of bordism class in $\mathfrak{T}_{*}^{\mathrm{PL}}(G / \mathrm{PL})$, and satisfies a product formula. Moreover, if the $x_{4 i+2}$ are suitably chosen, there exists a stable cohomology operation

$$
a=1+S q^{2}+S q^{2} S q^{2}
$$

such that if $k=a\left(\sum_{i \geq 0} x_{4 i+2}\right)$, and $f: M^{2 r} \rightarrow G / \mathrm{PL}$, then

$$
c(f)=w(M) \cdot f^{*}(k)[M] .
$$

Applying this to $f: P^{n} \rightarrow G / \mathrm{PL}$, we find that if $n=4 i-2$ or $4 i$, $i \geqq 2$, then for suitable $a_{n j} \in Z_{2}$,

$$
c(f)=\left[x_{4 i-2}\right]+\sum_{j<i} a_{n j}\left[x_{4 j-2}\right] .
$$

Finally, we will need the suspension. Let $n \geqq 5$, and let $Q^{n} \simeq P^{n}$. Then the double cover, $\tilde{Q}^{n}$, of $Q^{n}$ is PL-homeomorphic to $S^{n}$. Hence if we attach (by the projection map) the cone on $Q^{n}$ to $Q^{n}$, we have a closed $(n+1)$-manifold homotopy equivalent to $P^{n+1}$. We call this the suspension $\Sigma Q$; the definition stems from Browder and Livesay [1].

LEMMa 3. The following diagram is commutative:

$$
\begin{aligned}
& I_{4 i-2} \stackrel{\eta}{\rightarrow}\left[P^{4 i-2}, G / \mathrm{PL}\right] \\
& \downarrow \Sigma \quad r \uparrow \cong \searrow c_{4 i-2 \vartheta} \cong \\
& I_{4 i-1} \stackrel{\eta}{\rightarrow}\left[P^{4 i-1}, G / \mathrm{PL}\right] \stackrel{d \vartheta}{\rightarrow} Z_{2} \\
& \downarrow \Sigma \underset{\eta}{r \uparrow} \quad \nearrow c_{4 i} \vartheta \\
& I_{4 i} \stackrel{\eta}{\rightarrow}\left[P^{4 i}, G / \mathrm{PL}\right]
\end{aligned}
$$


The result follows from (c) and diagram chasing. A more direct proof has been found by Santiago López de Medrano [12].

TheOREM. We have the following bijections, for $i \geqq 1$,

$$
\begin{aligned}
I_{4 i+2} & \cong I_{4 i+1} \stackrel{\eta}{\cong}\left[P^{4 i+1}, G / \mathrm{PL}\right] \stackrel{X}{\cong} Z_{4} \oplus \sum_{8 \leq j \leq 2 i} Z_{2}, \\
(r, \tau): I_{4 i+8} & \cong I_{4 i+2} \oplus Z, \\
\left(r^{2},\left[x_{4 i+4}\right]\right): I_{4 i+4} & \cong I_{4 i+2} \oplus Z_{2} .
\end{aligned}
$$

The suspension $I_{4 i+3} \rightarrow I_{4 i+4}$ is the sum of the identity and the epimorphism $\boldsymbol{Z} \rightarrow \boldsymbol{Z}_{2}$.

Our computation of surgery obstructions has already determined $\operatorname{Im} \eta$ in all cases. Further, $\eta$ is injective on $I_{n}$ for $n$ even (since $\left.L_{n+1}\left(Z_{\overline{2}}\right)=0\right)$, and also if $n=4 i+1$, using the fact that $L_{4 i+2}(1)$ $\rightarrow L_{4 i+2}\left(Z_{2}^{+}\right)$is bijective and a known argument in the simply connected case. The same argument shows for $n=4 i+3$ that the image of $L_{4 i+4}(1) \rightarrow L_{4 i+4}\left(Z_{2}^{+}\right)$operates trivially on $I_{4 i+8}$, so we need only consider the quotient group, which is detected by the invariant $2 \sigma-\tilde{\sigma}$.

We now define a map $\tau: I_{4 i-1} \rightarrow Z$. Any homotopy projective space $Q^{4 i-1}$ determines naturally a bordism element $x \in \Omega_{4 i-1}^{\mathrm{PL}}\left(P_{\infty}(R)\right)$. This group is finite, so for some integer $N, N Q$ bounds $W^{4 i}$ say; let $\tilde{W}$ be the double cover of $W$ induced from $P_{\infty}(R)$. Since each component of $\partial W$ is a rational homology sphere, we can define a signature $\sigma(W)$, also $\sigma(\tilde{W})$. Since (by the Hirzebruch formula) the signature of a double covering of a closed oriented PL manifold is twice that of the manifold, $\tau(Q)=\{2 \sigma(W)-\sigma(\tilde{W})\} / 8 N$ depends only on $Q$. Then $\tau$ induces a bijection of each fibre of $\eta$ onto $Z$.

REMARK 1. Each of our invariants, reduced $\bmod 2$, is a topological invariant of $Q_{n}$. Except for $\tau$, this follows from the proofs (due, respectively, to Casson (unpublished) and Sullivan [8]) of the Hauptvermutung for 1-connected manifolds with $H_{3}(M ; Z)$ free of 2-torsion. For $\tau(\bmod 2)$ we use the suspension (which is defined topologically) and the last part of Theorem 4.

REMARK 2. For 4-manifolds, arguments analogous to the above show that there are at most $2 h$-cobordism classes of manifolds $Q_{4} \simeq P_{4}$; if there are 2 , they are distinguished by $y \eta$ (whose image will then be $Z_{2} \subset Z_{4}$ ). Even if the nontrivial class exists, it is not clear whether the double cover of any manifold in it will be $S^{4}$ itself.

For 3-manifolds, it is known that if $\widetilde{Q}_{3}=S^{3}$, then $Q_{3}=P_{8}$ (Livesay [3]). In lower dimensions, all becomes trivial. 
REMARK 3. Orientation-reversal of $P_{2 i+1}$ comes from reflection in $P_{2 i}$, and hence induces the identity on $\left[P_{2 i}, G / \mathrm{PL}\right]_{\approx}^{r 1}\left[P_{2 i+1}, G / \mathrm{PL}\right]$. However, it clearly alters the sign of $\tau$. Thus all our manifolds admit orientation-reversing PL-homeomorphisms except those of dimension $4 k+3$ with $\tau \neq 0$.

REMARK 4. Browder and Livesay have developed [1] an obstruction theory for the kernel and cokernel of $\Sigma$. In their exact sequences we have

$$
\begin{aligned}
& 0 \rightarrow I_{4 k} \stackrel{\Sigma}{\rightarrow} I_{4 k+1} \stackrel{f_{1}}{\rightarrow} Z_{2} \quad f_{1}=c \theta r \eta,
\end{aligned}
$$

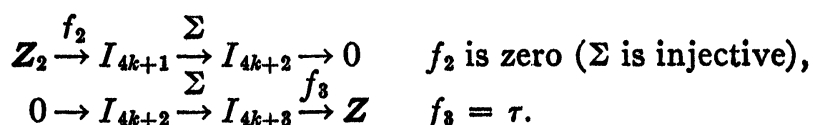

$$
\begin{aligned}
& \boldsymbol{Z} \stackrel{f_{4}}{\rightarrow} I_{4 k+3} \stackrel{\Sigma}{\rightarrow} I_{4 k+4} \rightarrow 0
\end{aligned}
$$

$f_{4}(n) Q=Q^{\prime}$, where $Q$ and $Q^{\prime}$ have the same normal invariant and $\tau\left(Q^{\prime}\right)=\tau(Q)+2 n$.

I am indebted to D. Sullivan and to F. Hirzebruch for supplying proofs of the result about $f_{3}$. Surjectivity of $f_{3}$ was also shown by S. López de Medrano[4].

REMarK 5. $P_{2 n+1}(R)$ is fibred over $P_{n}(C)$ with fibre $S^{1}$. The projecinduces an epimorphism

$$
\left[P_{n}(C), G / \mathrm{PL}\right] \rightarrow\left[P^{2 n+1}, G / \mathrm{PL}\right] .
$$

Given any (PL-) manifold $M^{2 n}$ homotopy equivalent to $P_{n}(C)$, we can take the induced fibration, and obtain an element of $I_{2 n+1}$. Moreover, it has been shown by Sullivan (for the $\boldsymbol{Z}_{2}$ case) and proved by Montgomery and Yang, and also by Hirzebruch (for the $Z$-case) that the Browder-Livesay obstruction above to desuspending the element of $I_{2 n+1}$ induces the obstruction to desuspending analogously $M^{2 n}$. Hence conversely, an element $Q_{2 n+1}$ of $I_{2 n+1}$ comes from the total space of such a fibration if and only if

$(n=2) . Q_{5}=P_{5}$.

$(n=3)$. The normal invariant of $Q_{7}$ is given by a certain function of $\tau\left(Q_{7}\right)$.

$(n=2 k, k>1)$. A mod 2 obstruction $z_{4 k}=\left[x_{4 k}\right]+$ lower terms vanishes.

$(n=2 k+1, k>1) . z_{4 k}$ equals the mod 2 reduction of $\tau$.

We cannot compute $z_{4 k}$ precisely since we have given no exact definition of the $x_{4 k}$.

REMARK 6. The problems of smooth free involutions on $S^{n}$ and on 
homotopy spheres remain unsolved. It is easy to obtain partial results -for example, S. López de Medrano has observed [12] that smooth homotopy projective spaces are determined modulo $\Theta_{n}(\partial \pi)$ by the smooth normal invariant and by $\tau$ (this follows easily from Lemma 2 above). We will not attempt to summarize all that is known, but refer to [2] for one of several known constructions which have yet to be fitted in with the viewpoint of this paper.

This paper was written while the author was visiting the Centro de Investigación y de Estudios Avanzados del I.P.N., Mexico.

\section{REFERENCES}

1. W. Browder and G. R. Livesay, Fixed point free involutions on homotopy spheres, Bull Amer. Math. Soc. 73 (1967), 242-245.

2. M. W. Hirsch and J. Milnor, Some curious involutions of spheres, Bull. Amer. Math. Soc. 70 (1964), 372-377.

3. G. R. Livesay, Fixed point free involutions on the 3-sphere, Ann. of Math. 72 (1960), 603-611.

4. S. L6pez de Medrano, Involutions of homotopy spheres and homology 3-spheres, Bull. Amer. Math. Soc. 73 (1967), 727-731.

5. J. W. Milnor, Microbundles and differentiable structures, Notes, Princeton University, 1961.

6. S. P. Novikov, Diffeomorphisms of simply connected manifolds, Dokl. Akad. Nauk SSSR 143 (1962), 1046-1049= Soviet Math. Dokl. 3 (1962), 540-543.

7. M. Spivak, Spaces satisfying Poincare duality, Topology 6 (1967), 77-102.

8. D. Sullivan, Triangulating and smoothing homotopy equivalences and homeomorphisms, Notes, Princeton University, 1967; also, On the Hauptvermutung for manifolds, Bull. Amer. Math. Soc. 73 (1967), 598-600.

9. C. T. C. Wall, Surgery of non-simply-connected manifolds, Ann. of Math. (2) 84 (1966), 217-276.

10. —- Poincare complexes. I, Ann. of Math. (2) 86 (1967), 213-245.

11. - Surgery of compact manifolds (to appear).

12. S. L6pez de Medrano, Some results on involutions of homotopy spheres, Proceedings of the Tulane conference on transformation groups, 1967 (to appear).

THE UNIVERSITY, LIVERPOOL 